\title{
A Comparative Study on the Efficacy of Covered Metal Stent and Plastic Stent in Unresectable Malignant Biliary Obstruction
}

\author{
Jae Myoung Choi', Jin Hong Kim¹, Soon Sun Kim¹, Jun Hwan Yu', \\ Jae Chul Hwang', Byung Moo Yoo', Sang Heum Park², Ho Gak Kim³, \\ Dong Ki Lee ${ }^{4}$, Kang Hyun Ko ${ }^{5}$, Kyo Sang Yoo ${ }^{6}$ and Do Hyun Park ${ }^{7}$
}

${ }^{1}$ Department of Gastroenterology, Ajou University School of Medicine, Suwon, ${ }^{2}$ Department of Internal Medicine, Soonchunhyang University College of Medicine, Cheonan, ${ }^{3}$ Department of Internal Medicine, Catholic University of Daegu School of Medicine, Daegu, ${ }^{4}$ Department of Internal Medicine, Yonsei University College of Medicine, Seoul, ${ }^{5}$ Digestive Disease Center, Bundang CHA Hospital, ${ }^{6}$ Department of Internal Medicine, Hallym University College of Medicine, Chuncheon, ${ }^{7}$ Department of Internal Medicine, Asan Medical Center, Seoul, Korea

Background/Aims: The placement of self expandable metal stent (SEMS) is one of the palliative therapeutic options for patients with unresectable malignant biliary obstruction. The aim of this study was to compare the effectiveness of a covered SEMS versus the conventional plastic stent.

Methods: We retrospectively evaluated 44 patients with unresectable malignant biliary obstruction who were treated with a covered SEMS (21 patients) or a plastic stent (10 Fr, 23 patients). We analyzed the technical success rate, functional success rate, early complications, late complications, stent patency and survival rate.

Results: There was one case in the covered SEMS group that had failed technically, but was corrected successfully using lasso. Functional success rates were $90.5 \%$ in the covered SEMS group and $91.3 \%$ in the plastic stent group. There was no difference in early complications between the two groups. Median patency of the stent was significantly prolonged in patients who had a covered SEMS (233.6 days) compared with those who had a plastic stent (94.6 days) $(p=0.006)$. During the follow-up period, stent occlusion occurred in 11 patients of the covered SEMS group. Mean survival showed no significant difference between the two groups (covered SEMS group, 236.9 days; plastic stent group, 222.3 days; $p=0.182$ ).

Conclusions: The patency of the covered SEMS was longer than that of the plastic stent and the lasso of the covered SEMS was available for repositioning of the stent.

Key Words: Malignant biliary obstruction; Self-expandable metal stent; Plastic stent

\section{INTRODUCTION}

Biliary stent insertion is performed in patients with unresectable malignant biliary ostruction as a palliative therapy to prevent early death due to deterioration of liver function or suppurative cholangitis. ${ }^{1-3}$ A plastic stent insertion had been the standard of the endoscopic biliary drainage, but this method was limited by the fact that it needs to be replaced

Received: December 5, 2010 Revised: February 18, 2012

Accepted: February 23, 2012

Correspondence: Jin Hong Kim

Department of Gastroenterology, Ajou University School of Medicine, 164 Worldcup-ro, Yeongtong-gu, Suwon 443-380, Korea

Tel: +82-31-219-6939, Fax: +82-31-219-5999, E-mail: jinhkim@ajou.ac.kr

(c) This is an Open Access article distributed under the terms of the Creative Commons Attribution Non-Commercial License (http://creativecommons.org/ licenses/by-nc/3.0) which permits unrestricted non-commercial use, distribution, and reproduction in any medium, provided the original work is properly cited. every 3 to 4 months due to the stent occlusion or migration. ${ }^{4}$ Self expandable metal stent (SEMS) with longer patency than the conventional plastic stent was developed to address this limitation and has been in use since $1989 .{ }^{5,6}$ An uncovered metal stent is advantageous in its low frequency of migration after the mesh was embedded with the tumor and the availability of using it in the intrahepatic bile duct; it is, however, associated with $20 \%$ to $40 \%$ incidence of early occlusion mainly due to the tumor ingrowth, ${ }^{7-9}$ and is not available for replacement or removal once it was placed inside the bile duct. $^{10,11}$

Covered SEMS was developed to address these limitations and to prolong the patency, but it also has limitations, such as higher frequency of migration compared with the uncovered metal stent ${ }^{12}$ and the difficulty of stent repositioning 
once inserted inside the bile duct. We performed this multicenter study to evaluate the clinical efficacy of a lasso-attached covered SEMS insertion to prevent tumor ingrowth in patients with malignant biliary obstruction and to compare the efficacy with the conventional 10 Fr plastic stent.

\section{MATERIALS AND METHODS}

\section{Patients}

This study was performed in patients who were diagnosed of having an extrahepatic biliary stricture due to a malignant tumor and received the endoscopic retrograde biliary drainage (ERBD) at 7 university hospitals in South Korea from January 2005 to July 2006. These patients were unresectable or refused a surgery. A retrospective analysis was performed in 21 patients who received the covered SEMS insertion and 23 patients who received the 10 Fr plastic stent insertion among those available for long-term follow-up.

Malignant biliary stricture was diagnosed base on sultrasonography, abdominal computed tomography (CT), magnetic resonance cholangiopancreatography, endoscopic retrograde cholangiopancreatography (ERCP), and histological findings. Patients with hilarcholangiocarcinoma, history of biliary stent insertion, concomitant duodenal obstruction, or past history of gastrointestinal operation were excluded from the study.

\section{Methods}

A 10-mm covered SEMS (Hanaro stent; M.I. Tech, Seoul, Korea), with lasso for location adjustment and silicone membrane for the prevention of tumor ingrowth, was used for the metal stent group (Fig. 1). A 10 Fr polyethylene plastic stent (Wilson-Cook Medical, Winston-Salem, NC, USA) was used for the plastic stent group.

Conventional side viewing duodenoscopes (JF 240V, TJF 240; Olympus Medical System Co., Tokyo, Japan) were used

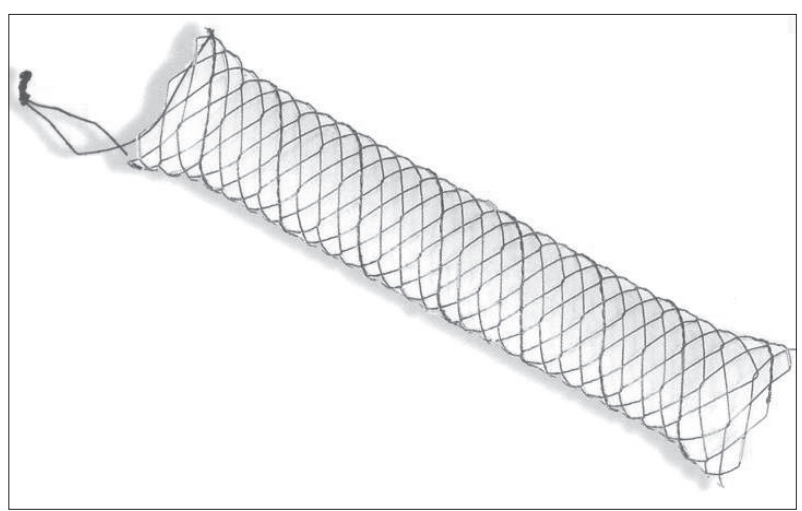

Fig. 1. New developed covered Hanaro stent. Covered biliary stent with lasso. Lasso enables to correct the stent position when it's misplaced. for ERCP in every patient. Endoscopic sphincterotomy (EST) was performed using a pull-type papillotome, and along the papillotome, a guide wire was inserted in the bile duct through the stricture site. The location and length of the stricture was assessed with the fluoroscopy, and then the stent was inserted along the guidewire. The Hanaro stent (M.I. Tech) was inserted across the stricture site into the extrahepatic bile duct, along the guidewire, until the distal end was exposed about $1 \mathrm{~cm}$ from the major papilla to the duodenum. For the plastic stent, a guidewire was first inserted into the bile duct across the stricture site for the following guiding catheter insertion into the bile duct, and then the plastic stent was pushed across the stricture site using the pusher catheter.

Technical success was defined when the stent was placed across the stricture and allowed the passing of the contrast media or the bile; while functional success was defined when the level of bilirubin decreased by $30 \%$ or more compared with the baseline level within a week or to a normal level within a month. Early or late complication was defined as any stent-related complication occurring within 30 days of or 30 days after the insertion, and the procedure-related death was defined as deaths due to complications associated with the EST or stent insertion. Functional failure of the stent was defined as signs of acute cholangitis, such as abdominal pain, jaundice or fever; increase of bilirubin level; or radiologic (such as abdominal CT or US) evidence of stent occlusion. Stent patency was defined as the period between the date of stent insertion and the date of functional failure or death by disease progression. We also determined the cause and period of stent functional failure as well as the cause and time to death.

\section{Statistical analysis}

Chi-square test was performed for comparison of categorical variables, and Student's t-test for continuous variables. Stent patency and patient survival were calculated according to the Kaplan-Meier analysis, and comparison of stent patency and survival between the two groups were analyzed by the log-rank test. A $p$-value $<0.05$ was considered significant. Statistical analysis was performed using SPSS version 12.0 (SPSS Inc., Chicago, IL, USA).

\section{RESULTS}

Among a total of 44 patients, 21 (10 males) were assigned to the Hanaro covered SEMS group and 23 (10 males) to the plastic stent group, and their mean age were $73.1 \pm 7.4$ years (range, 60 to 88 years) and $65.7 \pm 13.2$ years (range, 51 to 85 years), respectively. Biliary obstruction was caused by chol- 
angiocarcinoma in 12 patients, pancreatic cancer in 6 patients, gallbladder cancer in 2 patients and ampullary cancer in 1 patient of the covered SEMS group; and by cholangiocarcinoma in 9 patients, pancreatic cancer in 7 patients, gallbladder cancer in 4 patients and ampullary cancer in 3 patients of the plastic stent group, without a significant difference between the two groups (Table 1). The stricture site in the common bile duct was proximal in 2 patients, mid in 10 patients and distal in 9 patients of the covered SEMS group; proximal in 5 patients, mid in 4 patients and distal in 14 patients of the plastic stent group.

Technical success rates were $95.2 \%(20 / 21)$ in the covered SEMS group and $100 \%$ (23/23) in the plastic stent group, without any significant difference between the two groups ( $p=0.290$ ), except 1 case of technical failure due to the stent misplacement in the covered SEMS group, which was corrected using forceps to catch the lasso and achieved the functional success. Functional success rates were 90.5\% (19/21) in the covered SEMS group and $91.3 \%(21 / 23)$ in the plastic

Table 1. Baseline Characteristics of the Patients with Unresectable Malignant Biliary Obstruction

\begin{tabular}{lcc}
\hline & Hanaro stent $(n=21)$ & Plastic stent $(n=23)$ \\
\hline Age (yr, range) & $73.1 \pm 7.4(60-88)$ & $65.7 \pm 13.2(51-85)$ \\
Male/Female & $10 / 11$ & $10 / 13$ \\
Diagnosis & & \\
$\quad$ Bile duct cancer & 12 & 9 \\
Pancreatic cancer & 6 & 7 \\
Ampullary cancer & 1 & 3 \\
Gallbladder cancer & 2 & 4 \\
\hline
\end{tabular}

Values are presented as mean \pm SD. stent group, without a significant difference between the two groups ( $p=0.924)$. The length of the Hanaro covered SEMS was selected among 5, 6, 7, 8, 9 or 10 -cm length according to the length of the stricture, and the plastic stent among 5, 7, 9, 10 or 12 -cm length. The mean follow-up periods were 329.52 \pm 246.53 days (range, 4 to 908 days) in the covered SEMS group and $336.48 \pm 271.52$ days (range, 88 to 1,189 days) in the plastic stent group. Early complications occurred in 3 patients from each group. In the covered SEMS group, mild pancreatitis occurred in 1 patient, cholangitis in 1 patient and cholecystitis in 1, and all of them were recoverd with conservative treatment. In the plastic stent group, mild pancreatitis occurred in 1 patient and the patient was recovered with conservative treatment, but 1 patient of cholangitis required covered SEMS insertion and another patient of the cholangitis was treated with percutaneous transhepatic biliary drainage (PTBD) (Table 2).

The mean patency duration was significantly longer in the covered SEMS group (233.6 days; range, 21 to 660 days) compared with the plastic stent group (94.6 days; range, 4 to 549 days) ( $p=0.006$ ) (Table 2, Fig. 2).

The causes of stent occlusion in the covered SEMS group were bile sludge in 4 patients, tumor ingrowth in 4 patients, tumor overgrowth in 1 patient, stent migration in 1 patient and unknown origin in 1 patient. Among them, 9 patients were treated with plastic stent insertion inside the SEMS $(n=4)$, metal stent insertion $(n=4)$ and PTBD $(n=1)$. In the plastic stent group, the causes of stent occlusion were bile sludge in 6 patients, tumor overgrowth in 2 patients, late stent migration in 2 patients and unknown origin in 1 patient, which were treated with metal stent insertion after re-

Table 2. Outcome for Patients with Stent Placement

\begin{tabular}{lccc}
\hline & Hanaro stent & Plastic stent & $p$-value \\
\hline Technical success rate (\%) & $20 / 21(95.2)^{\mathrm{a})}$ & $23 / 23(100.0)$ & 0.290 \\
Functional success rate (\%) & $19 / 21(90.5)$ & $21 / 23(91.3)$ & \\
Early complication & & 1 & 2 \\
$\quad$ Pancreatitis & 1 & 0 & 0.924 \\
Cholangitis & 1 & $94.6(4-549)$ & \\
Cholecystitis & 1 & & \\
Mean stent patency (range), day & $233.6(21-660)$ & 0 & \\
Cause of obstruction & 4 & 2 \\
$\quad$ Bile clogging & 4 & 2 \\
Tumor ingrowth & 1 & 1 & 0.182 \\
Tumor overgrowth & 1 & $222.3(12-647)$ \\
Stent migration & $236.9(4-673)$ & & \\
Unknown & 4 & & \\
Mean survival (range), day & & & \\
\hline
\end{tabular}

a) One case that had failed technically, was repositioned successfully using lasso. 


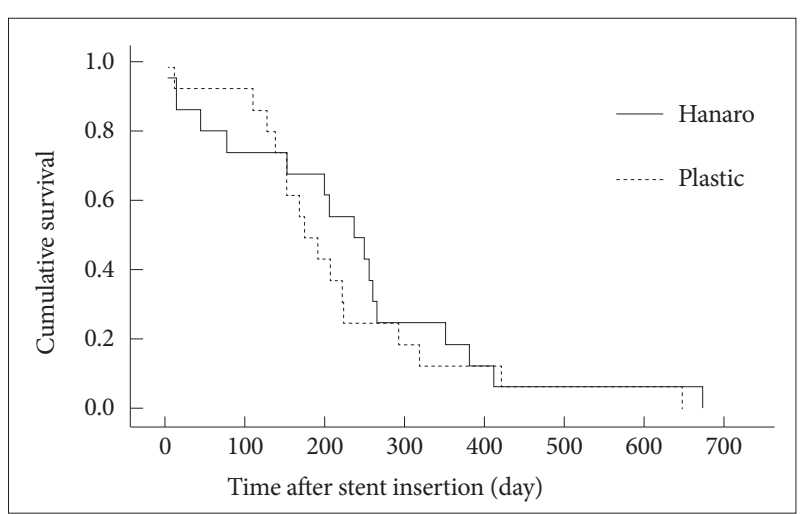

Fig. 2. Kaplan-Meier estimation of stent patency rates. Hanaro stent has significantly longer patency than plastic stent $(p=0.006)$.

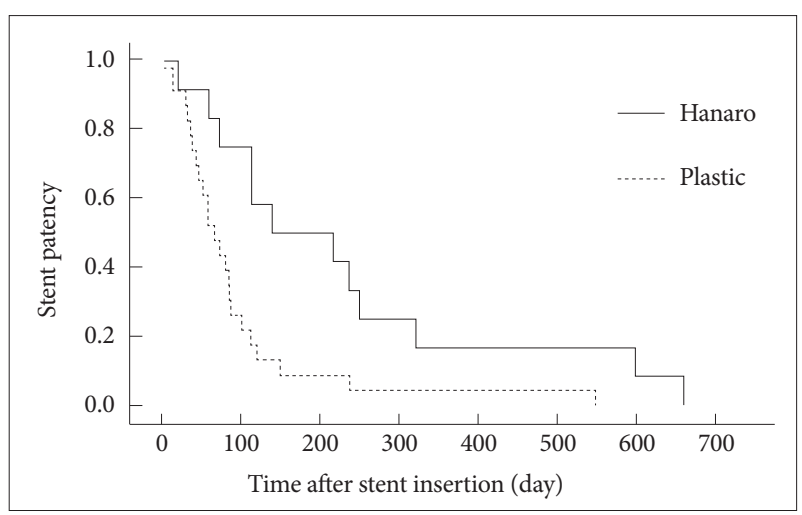

Fig. 3. Patients survival after stent insertion. The survival was not significantly different between two groups $(p=0.182)$.

moving the plastic stent $(n=6)$, re-insertion of the plastic stent $(n=3)$ and PTBD $(n=2)$ (Table 2).

Mean survival was 236.9 days (range, 4 to 673 days) in the Hanaro stent group and 222.3 days (range, 12 to 647 days) in the plastic stent group. There was no significant difference in the mean survival between the two groups ( $p=0.182$ ) (Fig. 3).

\section{DISCUSSION}

ERBD is effective in the prevention and treatment of cholangitis and enables physiologic drainage of the bile, making it a suitable palliative therapy for patients with unresectable malignant biliary obstruction. ${ }^{2,13}$ The indications of ERBD include malignant biliary obstruction due to pancreatic cancer or cholangiocarcinoma, suppurative cholangitis, common bile duct stone impaction, bile duct stricture after surgery, and obstructive jaundice concomitant with chronic pancreatitis. In malignant biliary obstruction, the placement of biliary plastic stent rapidly improves the jaundice and allows semi-permanent biliary drainage in unresectable cases. However, the plastic stent is not permanent and requires replacement or additional stent insertion in several months when the occlusion occurs. Efforts have been made to reduce the stent occlusion, such as by decreasing bacterial adhesion inside the stent or by changing the material or structure-size and shape, that is-of the stent, ${ }^{14}$ without an apparent success yet.

Plastic stent is not expensive and replaceable, but requires frequent replacement due to the stent migration or frequent occlusion leading to the recurrence of obstructive jaundice or cholangitis. ${ }^{8,15}$ Metal stent was developed in 1989 to overcome these limitations and has been in use since then. ${ }^{5,6}$ SEMS is associated with less occlusion and cholangitis and shorter duration of hospital stay compared with the plastic stent. ${ }^{7.8}$ The plastic stent induces occlusion mostly by the formation of biliary sludge. The metal stent, however causes occlusion mostly by the tumor ingrowth, where the tumor grows through the gaps of the stent mesh; by the tumor overgrowth, where the tumor grows to the upper and lower space of the stent; and by epithelial hyperplasia inside the stent. ${ }^{16,17}$ The rate of metal stent occlusion due to the tumor ingrowth or overgrowth after some time, has been reported variously from $7 \%$ to $42 \% .5,10,18-20$

According to a prospective trial by Davids et al., ${ }^{7}$ the early efficacy of drainage by the plastic stent and the metal stent are not significant different at $95 \%$ and $96 \%$, respectively. This study also revealed no difference of the early efficacy by the materials of the stents, with the polyethylene plastic stent and the Hanaro covered SEMS respectively showing 91.3\% $(21 / 23)$ and $90.5 \%$ (19/21) of functional success rates. Given the fact that a stent of 7 Fr or more is physiologically sufficient to induce biliary drainage, ${ }^{21}$ this lack of difference in the early efficacy of drainage between the plastic stent group and the Hanaro covered SEMS group in our study may come from using $10 \mathrm{Fr}$ plastic stents. The time to biliary stent occlusion of a 10 Fr plastic stent has been reported average 134 to 164 days. ${ }^{22,23}$ Davids et al. ${ }^{7}$ reported stent patency of 273 days for metal stent (Wallstent; Schneider, Minneapolis, MN, USA) and 126 days for polyethylene plastic stent in distal malignant biliary obstruction. Our findings also showed 233.6 days and 94.6 days of mean stent patency for the Hanaro covered SEMS group and the plastic group, respectively.

The advantage of the SEMS is that the stent diameter can be increased remarkably to allow early sufficient drainage, the duration of patency is longer than the plastic stent, and the frequency of migration is low. But several disadvantages of the SEMS have been also pointed out, including the difficulty of insertion technique compared with that of the plastic stent, ${ }^{24}$ the possibility of injuries such as bleeding or perforation in the duodenum and the papilla, ${ }^{25}$ high cost, inability to remove once placed, and the possibility of stent occlusion due to the tumor ingrowth or overgrowth. ${ }^{26}$ Despite the development of covered SEMS, using a thin layer of polyurethane, silicon, polytetrafluoroethylene or Gore-Tex to cover 
SEMS ( $30 \mathrm{Fr}$ ), to prevent functional failure due to the tumor ingrowth or overgrowth, tumor ingrowth is still reported to occur with the membrane covered metal stent. ${ }^{27-30}$ Suh et al. ${ }^{31}$ reported, in a study of 59 patients who were diagnosed with extrahepatic biliary stricture due to the malignant tumor, that the tumor ingrowth was the cause of the stent functional failure in $6.6 \%(2 / 29)$ of the covered SEMS group compared to $24 \%(7 / 30)$ of the uncovered SEMS group, suggesting that the covered SEMS may prevent the tumor ingrowth efficiently. The functional failure of the Hanaro covered SEMS in our study was due to stent occlusion in 9 patients and of migration in 1 patient (late stent migration); among these 9 patients of occlusion, 4 patients (44.4\%) were associated with the tumor ingrowth, 1 patient with the tumor overgrowth, and the remaining 4 patients with the biliary sludge, reflecting that the covering membrane of the SEMS cannot prevent the tumor ingrowth completely. Tumor ingrowth in the covered SEMS may occur when the membrane was damaged during the stent insertion, by the bile or biliary stone, or by bacterial infection, but little data are available regarding the exact cause of tumor ingrowth.

Early complications occurring during the stent insertion or within 2 weeks after the insertion include cholangitis, cholecystitis, perforation, bleeding, and acute pancreatitis, at $6.4 \%$ to $26.3 \%$ of incidence, among which cholangitis occurring most frequently. ${ }^{22}$ Davids et al. ${ }^{7}$ reported that $11 \%$ of the plastic stent group and $12 \%$ of the metal stent group were associated with the early (within a week after the stent insertion) complications, which is similar to our results of $12.5 \%$ (3/23) for the plastic stent group and $14.3 \%(3 / 21)$ for the Hanaro covered SEMS group. Isayama et al. ${ }^{32}$ reported that the membrane attached to a stent often blocks the orifice of the cystic duct or the pancreatic duct causing cholecystitis or pancreatitis. In this study, there were 3 cases of early complications in the both groups, repectively. In the Hanaro covered SEMS group, there were pancreatitis in 1 patient, cholangitis in 1 patient and cholecystitis in 1 patient, all of them were recovered with conservative treatment. In the plastic stent group, pancreatitis occurred in 1 patient and cholangitis in 2 patients; the former was recovered with conservative treatment, while each of the patients with cholangitis required SEMS insertion and PTBD, respectively.

There is still a controversy in what type of stent should be used for ERBD in terms of cost-effectiveness. Prat et al. ${ }^{33}$ reported 3.2 months of survival for $\geq 30 \mathrm{~mm}$ of tumor diameter and 6.6 months of survival for $\leq 30 \mathrm{~mm}$ of tumor diameter in 101 patients with unresectable malignant biliary obstruction. Therefore, they insisted that the metal stent may be more effective than the plastic stent when the tumor diameter is 30 $\mathrm{mm}$ or less in unresectable malignant biliary obstruction. ${ }^{33}$
Schmassmann et al. ${ }^{17}$ also reported that the metal stent was more effective than the plastic stent in patients with more than 6 to 9 months of life expectancy.

Cahen et al. ${ }^{34}$ emphasized that modification of the SEMS design was necessary for convenient removal and repositioning of the stent. The lasso attached on the covered SEMS in our study allowed functional success in 1 case of technical failure during the insertion by enabling the stent repositioning at ease.

In conclusion, the placement of the covered SEMS with longer stent patency seemed to be more effective than that of the plastic stent in patients with unresectable malignant biliary obstruction who their life expectancy are estimated to be longer than 6 to 9 months based on their general condition, the presence of comorbidity and biliary stricture diameter. The lasso of the covered SEMS seemed to be helpful in repositioning of the stent to achieve functional success, although this issue needs to be further studied in prospective studies with more cases.

\section{Conflicts of Interest}

The authors have no financial conflicts of interest.

\section{REFERENCES}

1. Gundry SR, Strodel WE, Knol JA, Eckhauser FE, Thompson NW. Efficacy of preoperative biliary tract decompression in patients with obstructive jaundice. Arch Surg 1984;119:703-708.

2. Andersen JR, Sørensen SM, Kruse A, Rokkjaer M, Matzen P. Randomised trial of endoscopic endoprosthesis versus operative bypass in malignant obstructive jaundice. Gut 1989;30:1132-1135.

3. Shepherd HA, Royle G, Ross AP, Diba A, Arthur M, Colin-Jones D. Endoscopic biliary endoprosthesis in the palliation of malignant obstruction of the distal common bile duct: a randomized trial. Br J Surg 1988;75:1166-1168.

4. Gilbert DA, DiMarino AJ Jr, Jensen DM, et al. Status evaluation: biliary stents. American Society for Gastrointestinal Endoscopy. Technology Assessment Committee. Gastrointest Endosc 1992;38:750-752.

5. Huibregtse K, Cheng J, Coene PP, Fockens P, Tytgat GN. Endoscopic placement of expandable metal stents for biliary strictures: a preliminary report on experience with 33 patients. Endoscopy 1989;21:280282.

6. Irving JD, Adam A, Dick R, Dondelinger RF, Lunderquist A, Roche A. Gianturco expandable metallic biliary stents: results of a European clinical trial. Radiology 1989;172:321-326.

7. Davids PH, Groen AK, Rauws EA, Tytgat GN, Huibregtse K. Randomised trial of self-expanding metal stents versus polyethylene stents for distal malignant biliary obstruction. Lancet 1992;340:1488-1492.

8. Knyrim K, Wagner HJ, Pausch J, Vakil N. A prospective, randomized, controlled trial of metal stents for malignant obstruction of the common bile duct. Endoscopy 1993;25:207-212.

9. Wagner HJ, Knyrim K, Vakil N, Klose KJ. Plastic endoprostheses versus metal stents in the palliative treatment of malignant hilar biliary obstruction. A prospective and randomized trial. Endoscopy 1993;25:213218.

10. Bezzi M, Orsi F, Salvatori FM, Maccioni F, Rossi P. Self-expandable nitinol stent for the management of biliary obstruction: long-term clinical results. J Vasc Interv Radiol 1994;5:287-293.

11. Mueller PR. Metallic endoprostheses: boon or bust? Radiology 1991; 
179:603-605.

12. Kanasaki S, Furukawa A, Kane T, Murata K. Polyurethane-covered nitinol Strecker stents as primary palliative treatment of malignant biliary obstruction. Cardiovasc Intervent Radiol 2000;23:114-120.

13. Luman W, Cull A, Palmer KR. Quality of life in patients stented for malignant biliary obstructions. Eur J Gastroenterol Hepatol 1997;9: 481-484.

14. Coene PP, Groen AK, Cheng J, Out MM, Tytgat GN, Huibregtse K. Clogging of biliary endoprostheses: a new perspective. Gut 1990;31: 913-917.

15. Rey JF, Maupetit P, Greff M. Experimental study of biliary endoprosthesis efficiency. Endoscopy 1985;17:145-148.

16. O'Brien S, Hatfield AR, Craig PI, Williams SP. A three year follow up of self expanding metal stents in the endoscopic palliation of longterm survivors with malignant biliary obstruction. Gut 1995;36:618-621.

17. Schmassmann A, von Gunten E, Knuchel J, Scheurer U, Fehr HF, Halter F. Wallstents versus plastic stents in malignant biliary obstruction: effects of stent patency of the first and second stent on patient compliance and survival. Am J Gastroenterol 1996;91:654-659.

18. Hausegger KA, Kleinert R, Lammer J, Klein GE, Fluckiger F. Malignant biliary obstruction: histologic findings after treatment with selfexpandable stents. Radiology 1992;185:461-464.

19. Lee MJ, Dawson SL, Mueller PR, Krebs TL, Saini S, Hahn PF. Palliation of malignant bile duct obstruction with metallic biliary endoprostheses: technique, results, and complications. J Vasc Interv Radiol 1992;3:665-671.

20. Moesch C, Sautereau D, Cessot F, et al. Physicochemical and bacteriological analysis of the contents of occluded biliary endoprostheses. Hepatology 1991;14:1142-1146.

21. Shim CS. [The endoscopic therapy of biliary tract disease]. Korean J Gastrointest Endosc 1989;9:127-129.

22. Classen M, Hagenmuller F. Biliary drainage. Endoscopy 1983;15 Suppl 1:221-229.

23. Wurbs D, Phillip J, Classen M. Experiences with the long standing na- sobiliary tube in biliary diseases. Endoscopy 1980;12:219-223.

24. Bethge N, Wagner HJ, Knyrim K, et al. Technical failure of biliary metal stent deployment in a series of 116 applications. Endoscopy 1992; 24:395-400.

25. Ee $\mathrm{H}$, Laurence $\mathrm{BH}$. Haemorrhage due to erosion of a metal biliary stent through the duodenal wall. Endoscopy 1992;24:431-432.

26. Huibregtse K, Carr-Locke DL, Cremer M, et al. Biliary stent occlusion: a problem solved with self-expanding metal stents? European Wallstent Study Group. Endoscopy 1992;24:391-394.

27. Born P, Neuhaus $H$, Rosch T, et al. Initial experience with a new, partially covered Wallstent for malignant biliary obstruction. Endoscopy 1996;28:699-702.

28. Rossi P, Bezzi M, Salvatori FM, Panzetti C, Rossi M, Pavia G. Clinical experience with covered wallstents for biliary malignancies: 23-month follow-Up. Cardiovasc Intervent Radiol 1997;20:441-447.

29. Shim CS, Lee YH, Cho YD, et al. Preliminary results of a new covered biliary metal stent for malignant biliary obstruction. Endoscopy 1998; 30:345-350.

30. Thurnher SA, Lammer J, Thurnher MM, Winkelbauer F, Graf O, Wildling R. Covered self-expanding transhepatic biliary stents: clinical pilot study. Cardiovasc Intervent Radiol 1996;19:10-14.

31. Suh JH, Song SY, Park SW, et al. A new covered biliary metal stent versus uncovered wallstent for malignant biliary obstruction. Korean J Gastrointest Endosc 2001;23:93-99.

32. Isayama H, Kawabe T, Nakai Y, et al. Cholecystitis after metallic stent placement in patients with malignant distal biliary obstruction. Clin Gastroenterol Hepatol 2006;4:1148-1153.

33. Prat F, Chapat O, Ducot B, et al. A randomized trial of endoscopic drainage methods for inoperable malignant strictures of the common bile duct. Gastrointest Endosc 1998;47:1-7.

34. Cahen DL, Rauws EA, Gouma DJ, Fockens P, Bruno MJ. Removable fully covered self-expandable metal stents in the treatment of common bile duct strictures due to chronic pancreatitis: a case series. Endoscopy 2008;40:697-700. 